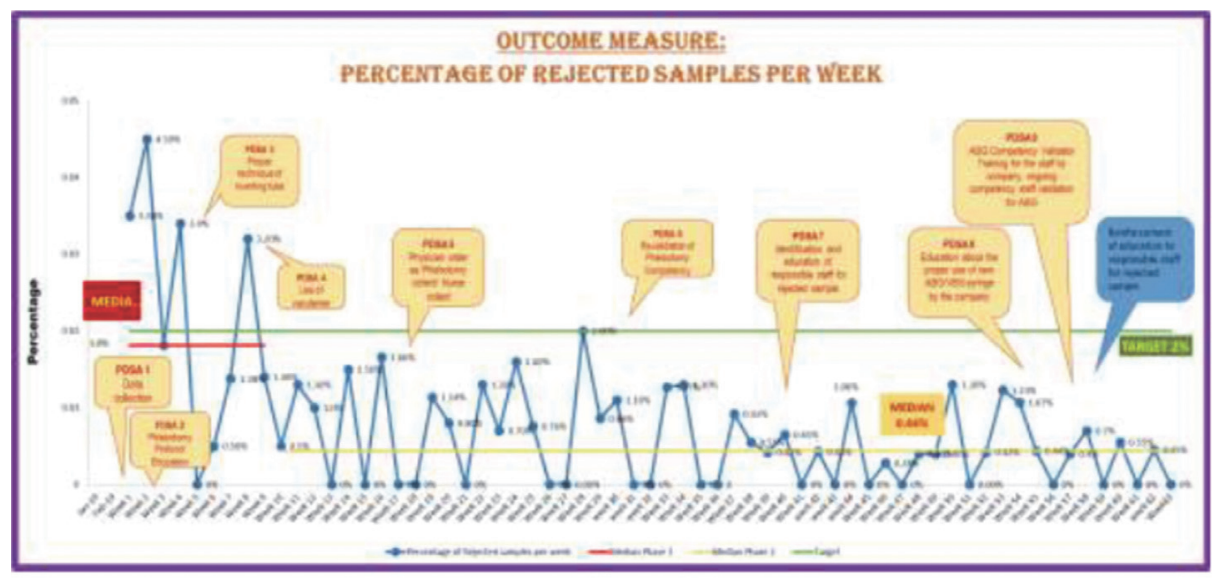

$24 \%$ decrease in

rejected samples
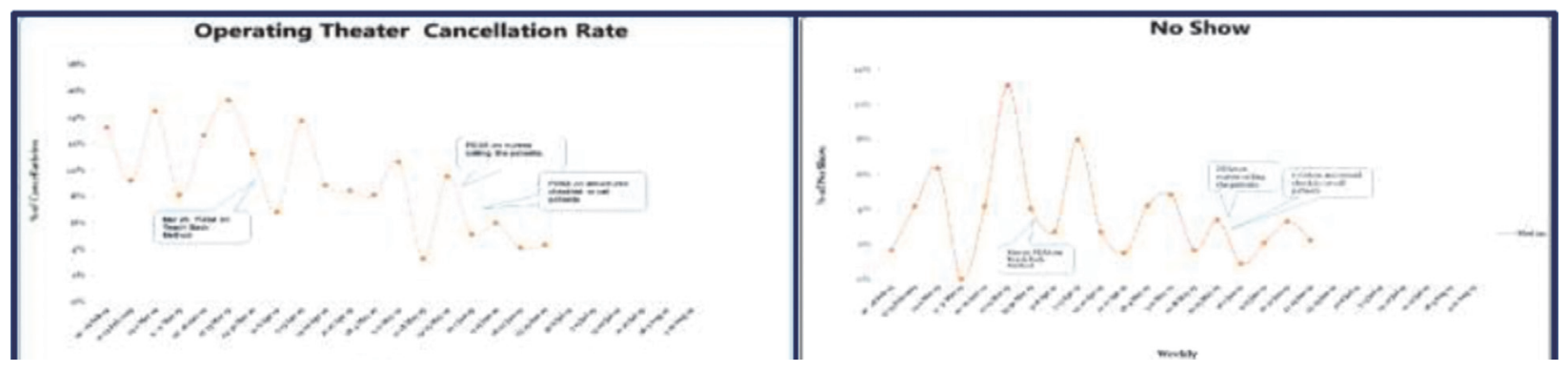

Abstract 3 Figure 7

transformational outcomes in harm reduction, utilization of resource and improved team culture.

Objectives Apply Macro to Meso-system approach to Value Improvement to achieve national system wide strategic objectives. Apply Value Improvement methods to apply innovative approaches to build new evidence to reduce waste, harm and cost whilst improving multi-disciplinary team work and joy in work. Learn how to successfully scale up and spread value Improvement across a health system (figure 1).

Methods Value improvement focuses on improving performance, capacity \& finance measures by achieving reliable care delivery eg early discharge, reduced rejected samples, reduction in falls, reduction in waste and consumable costs, whilst increasing joy in work. It has 3 components: Box Score, Visual Management Board and Weekly Multi-Disciplinary 15 minute Huddles. It was initially piloted, evaluated, spread to $2 / 6$ units and 35 teams across the healthcare system. The audience would apply some inter- active learning to understand all key concepts and how to apply in their own setting

Results $24 \%$ decrease in rejected samples. Patient discharge before $1 \mathrm{pm}$ reached $90 \%$ \& sustained. 24\% reduction in cost of consumables (figures 4 , and 6). Improve theatre utilization to $80 \%$ (figure 7 ) and sustaining. 36\% reduction in average waiting time in recovery (figures 2, 3, and 5).

Conclusions The Value Improvement approach offers a systematic method for continuously improving and innovating on a daily/weekly basis to achieve demonstrable results. This changes team culture as the team work to improve multiple projects to meet the needs of our patients, family and staff. By focusing attention and reporting out each week numerous improved measures created a way to engage staff in continuous innovation and improvement.

\section{PROVIDING THE OPTIMAL RESIDENT EDUCATION EXPERIENCE DURING FAMILY CENTERED ROUNDS}

Sarah Calardo, Nadia Chaudhry-Waterman, Peter Janzen, David Kling, Lesya Tomlinson, Darshita Bhatia, Candyce Greene, Stephen Moses, Amy Weis, Courtney Port. Inova Children's Hospital, USA

\subsection{6/bmjoq-2020-IHI.4}

Background Family Centered Rounds (FCR) allow the medical team to partner with patients and families in medical decisionmaking, improving patient communication and safety. Our children's hospital recently increased FCR utilization by implementing geographic-based teams. A balancing measure demonstrated that while the majority of respondents agreed that FCR provided the best patient care, only $20 \%$ felt FCR provided the best educational experience.

Objectives Increase the percentage of residents with a positive perception of the educational experience on FCR from $20 \%$ to $80 \%$ in 6 months.

Methods The Model for Improvement and sequential PDSA cycles were utilized. A needs assessment was conducted to identify barriers to education (figure 1). The FCR process was standardized to include formal presentations outside patient rooms, addressing several educational experiences previously reported as being negatively impacted by FCR. Rounding duration and the percentage of patients receiving FCR were measured daily, while monthly surveys tracked resident perception of the educational experience. Family satisfaction and comprehension surveys were collected quarterly.

Results Residents who perceive FCR as a positive educational experience improved from $20 \%$ to $75 \%(\mathrm{~N}=8,6)$ (figure 2). Patients receiving FCR remained above $80 \%(\mathrm{~N}=267,178$, 209). $100 \%(\mathrm{~N}=14)$ of families understood their care plans 
and remained satisfied with the amount of information presented (figures 3 and 4). Unfortunately, rounds frequently exceeded the allotted time, increasing from $37 \%$ to $50 \%$ $(\mathrm{N}=7,6,10)$ (figure 5).

Conclusions Hybridization of FCR to include formal presentations resulted in 55\% more residents agreeing that FCR provided the best educational experience, while preserving family

\section{NEEDS ASSESSMENT:}

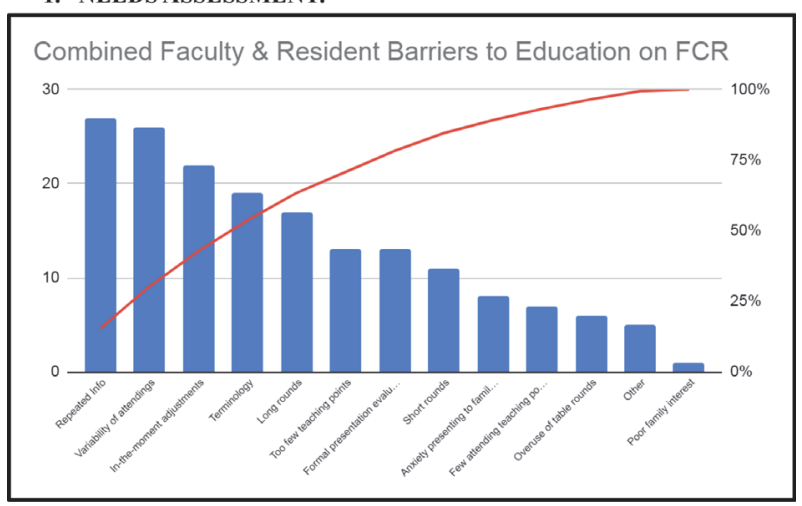

Abstract 4 Figure 1 Needs assesment: faculty and resident perceived barriers to education on FCR prior to QI project initiation

2. OUTCOME MEASURE:

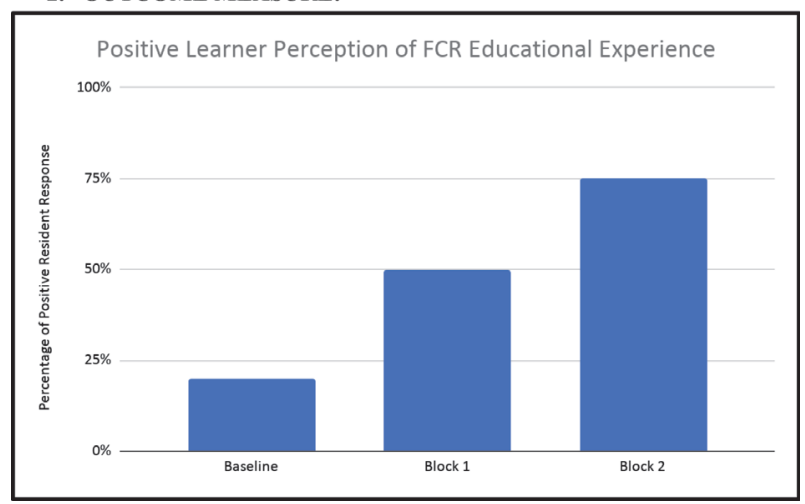

Abstract 4 Figure 2 Outcome measure: resident perception of FCR educational experience

3. BALANCING MEASURES:

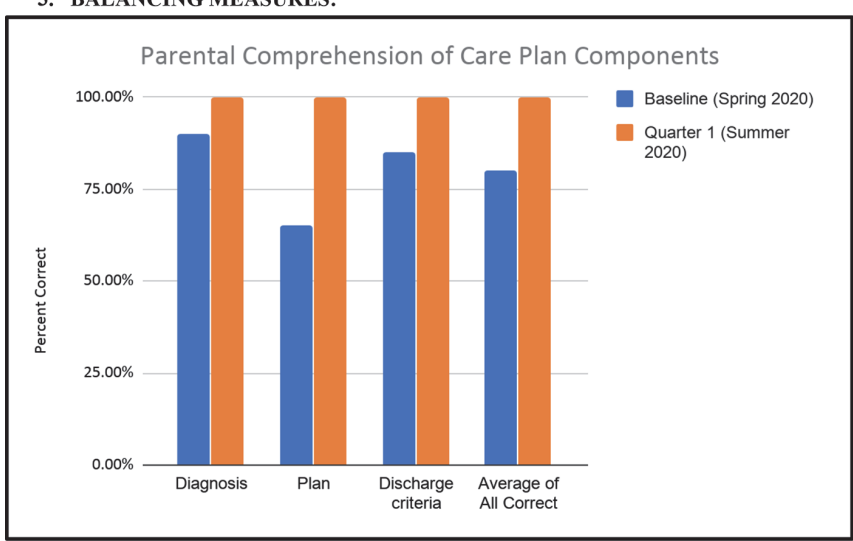

Abstract 4 Figure 3 Balancing measure: parental comprehension of diagnosis, plan, and discharge criteria

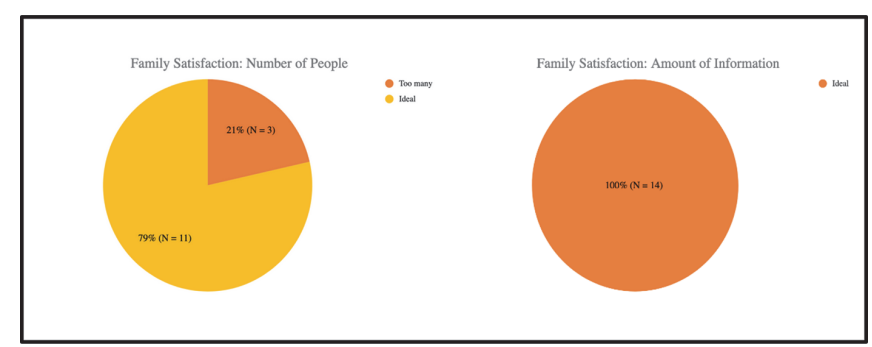

Abstract 4 Figure 4 Balancing measure: parental satisfication with FCR elements: number of people in the patient's room (during the COVID-19 pandemic) and the amount of information shared during FCR

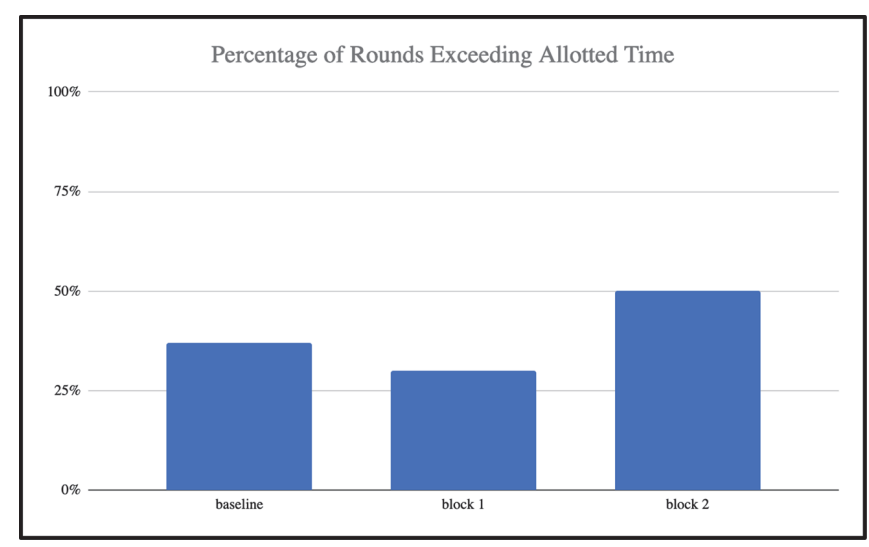

Abstract 4 Figure 5 Balancing measure: percentage of hospitalist rounds exceeding allotted time

satisfaction and comprehension. Results may be generalizable to similarly sized residency programs. Future interventions will target barriers and negatively impacted educational activities identified upon re-evaluation of the new FCR structure.

\section{REDUCING ACUTE HOSPITALIZATION LENGTH OF STAY AFTER TOTAL KNEE ARTHROPLASTY: A QUALITY IMPROVEMENT STUDY}

${ }^{1}$ Yehoshua Gleicher, 'Naveed Siddiqui, 'Y Yusuke Mazda, 'David Backstein, ${ }^{2} J o h n$ Matelski, ${ }^{3}$ Jesse Wolfstadt. 'Sinai Health System, Canada; ${ }^{2}$ University Health Network, Canada; ${ }^{3}$ Mount Sinai Hospital, University of Toronto, Canada

\subsection{6/bmjoq-2020-IHI.5}

Background The introduction of bundled funding for total knee arthroplasty (TKA) has motivated hospitals to improve the quality of care while minimizing costs. We identified LOS and the percentage of patients discharged to inpatient rehabilitation for primary TKA patients as potential targets for reducing costs, improving the quality of patient care, and ultimately improving our performance within the bundled payment model.

Objectives The aim of our quality improvement project was to reduce the acute hospitalization length of stay (LOS) to less than two days and decrease the percentage of TKA patients discharged to inpatient rehabilitation using an enhanced recovery after surgery (ERAS) bundle.

Methods This study used a before-and-after design. The preintervention period was January - December 2017 and the post-intervention period was January 2018 - August 2019. A 